\title{
Análise Exploratória das Informações sobre Estadiamento nas Autorizações de Procedimentos de Alta Complexidade no Brasil e Regiões no Período 2010-2014 Exploratory Analysis of the Information on Staging in the Authorizations of High Complexity Procedure in Brazil and Regions in the Period 2010-2014 Análisis Exploratorio de las Informaciones sobre Estadificación en las Autorizaciones de Procedimientos de Alta Complejidad en Brasil y Regiones en el Período 2010-2014
}

\author{
Adriana Tavares de Moraes Atty'; Jeane Glaucia Tomazelli; Maria Beatriz Kneipp Dias ${ }^{3}$
}

\section{Resumo}

Introduçáo: A radioterapia e a quimioterapia, modalidades terapêuticas para o tratamento do câncer no sistema público de saúde, exigem liberação da Autorização de Procedimento Ambulatorial de Alta Complexidade (APAC), a qual reúne informaçóes importantes sobre os pacientes e sobre os estabelecimentos de saúde. O objetivo deste estudo foi realizar uma análise exploratória das informaçóes sobre o estádio dos casos de câncer de mama. Método: Estudo exploratório, descritivo, sobre o estadiamento de câncer de mama, no Brasil e Regiôes, informado nas APAC de quimioterapia, no período de janeiro de 2010 a dezembro de 2014. Foram consideradas as variáveis: faixa etária, estadiamento, código de autorização da APAC, Cartão Nacional de Saúde (CNS) e Região do país do estabelecimento de saúde. Os dados foram obtidos por meio do DATASUS. Resultados: Total de 2.211.456 APAC iniciais referentes a 280.379 CNS. APAC com CNS repetidos corresponderam a 98,6\%, sendo a razão APAC/CNS de 8,7. Destas, 18,1\% tiveram alteração no estadiamento. Conclusáo: $\mathrm{O}$ estadiamento clínico estabelecido no início do tratamento deveria permanecer inalterado ao longo da vida do paciente, segundo a norma que orienta o preenchimento da APAC. Contudo, seria de grande valia ao monitoramento dos casos se fosse possível registrar o avanço da doença e, consequentemente, do estadiamento. Não foi possível afirmar se as mudanças observadas no estadiamento representavam evolução da doença ou erro de informaçáo. Sendo a APAC uma fonte de informaçáo útil ao planejamento e ao monitoramento dos serviços de saúde, vale zelar pela qualidade da informação sem negar a necessidade de aprimoramento do sistema.

Palavras-chave: Estadiamento de Neoplasias; Sistema Único de Saúde; Sistemas de Informação em Saúde.

\footnotetext{
${ }^{1}$ Mestre em Saúde Pública pela Fundação Oswaldo Cruz (Fiocruz). Tecnologista em C\&T pelo Instituto Nacional de Câncer José Alencar Gomes da Silva (INCA). Rio de Janeiro (RJ), Brasil. E-mail: aatty@inca.gov.br.

${ }^{2}$ Doutora em Saúde Coletiva pelo Instituto de Medicina Social pela Universidade Estadual do Rio de Janeiro (IMS/Uerj). Tecnologista em C\&T pelo INCA. Rio de Janeiro (RJ), Brasil. E-mail: jtomazelli@inca.gov.br.

${ }^{3}$ Mestre em Epidemiologia pela Fiocruz. Tecnologista em C\&T pelo INCA. Rio de Janeiro (RJ), Brasil. E-mail: mdias@inca.gov.br.

Endereço para correspondência: Adriana Tavares de Moraes Atty. Rua Marquês de Pombal, 125, 70 andar - Centro. Rio de Janeiro (RJ), Brasil.

CEP 20230-240.E-mail: aatty@inca.gov.br.
} 


\section{INTRODUÇÃO}

O tratamento do câncer admite três possibilidades terapêuticas: a cirurgia, a radioterapia e a quimioterapia; nesta última, incluídas as modalidades de hormonioterapia, terapia-alvo e imunoterapia. Estas podem ser utilizadas isoladamente ou em combinação a depender do tipo de tumor, localização, estadiamento e características físicas e emocionais do paciente ${ }^{1}$.

Somente hospitais habilitados em oncologia podem oferecer tratamento para neoplasias malignas na rede pública de saúde do Brasil. Esses estabelecimentos podem ser habilitados para oferecer as três modalidades terapêuticas ou ao menos cirurgia e quimioterapia ${ }^{2}$.

A realização do tratamento quimioterápico e radioterápico nesses hospitais estará sujeita à liberação da Autorizaçáo de Procedimento Ambulatorial de Alta Complexidade (APAC) mediante o preenchimento do laudo para solicitaçãolautorização de procedimentos ambulatoriais, formulário padronizado que contém informaçóes sobre o paciente, o tratamento indicado e sobre o profissional responsável ${ }^{3}$.

As APAC podem ser registradas com três códigos: 1 (inicial), 2 (continuidade) e 3 (única). Se o tratamento admitir continuidade, com validade de três meses, a autorização será registrada com o código 1 ou 2, sendo o código 1 para autorização apresentada na primeira competência e o código 2 para autorizaçáo apresentada no segundo ou terceiro mês. Se o tratamento não admitir continuidade, poderá ser utilizado o código 1 (a autorização valerá apenas para um mês) ou o 3 (a autorização valerá por três meses) $)^{3}$.

A APAC dispõe de informaçóes epidemiológicas e assistenciais, tais como: Classificação Internacional da Doença (CID), tipo do tumor, estadiamento, envolvimento de linfonodos, procedimento a ser realizado, e informaçôes sobre o paciente, tais como: Cartão Nacional de Saúde (CNS), data de nascimento, sexo, raça/ cor e município de residência e sobre o estabelecimento de saúde executante como, por exemplo, o número do Cadastro Nacional de Estabelecimentos de Saúde (CNES).

Entre as informaçóes registradas na APAC, destaca-se o estadiamento do tumor. Este deve ser estabelecido assim que o diagnóstico de câncer é confirmado e é fundamental para determinar o plano terapêutico e avaliar o prognóstico ${ }^{1}$. Segundo o manual de bases técnicas da $\mathrm{APAC}^{3}$, o estadiamento clínico é imutável, sendo estabelecido ao diagnóstico e antes de qualquer terapêutica. Assim sendo, a informação sobre o estádio clínico não deve ser modificada ainda que a extensão do tumor se modifique ao longo do tempo ou reapareça após tratamento ${ }^{4}$.
Os estádios mantêm uma relação de coerência com o sistema de classificação de tumores malignos (TNM), que possibilita efetuar a classificação do estadiamento em clínica (TNM ou cTNM), e patológica (pTNM). Enquanto o estadiamento clínico é o realizado no momento do diagnóstico visando a selecionar e avaliar o tratamento, o estadiamento patológico corresponde àqueles efetuados a partir de análise histopatológica pós-cirúrgica para avaliar o prognóstico ${ }^{4}$.

De acordo com a classificação TNM, o carcinoma in situ é categorizado como estádio 0; tumores restritos ao órgáo de origem como estádios I e II; tumores disseminados localmente como estádio III; e tumores com metástases distantes do órgão de origem como estádio IV ${ }^{4}$.

Os dados da APAC são registrados no Sistema de Informação Ambulatorial do Sistema Único de Saúde (SIA/SUS) que, muito embora se caracterize por ser um sistema de cunho eminentemente administrativo ${ }^{5}$ e financeiro, presta-se à vigilância, monitoramento e avaliaçáo pela natureza dos dados ali armazenados.

Este estudo teve por objetivo realizar uma análise exploratória das informaçóes sobre estadiamento registrada nas APAC de quimioterapia para o tratamento do câncer de mama por meio do relacionamento das autorizaçôes com os CNS.

\section{MÉTODO}

Trata-se de um estudo exploratório, descritivo, sobre o estadiamento de câncer de mama, informado nas APAC em oncologia e apresentados pelos estabelecimentos de saúde habilitados para o tratamento do câncer no SUS, no Brasil, no período entre janeiro de 2010 a dezembro de 2014.

As informaçôes de quimioterapia foram obtidas da APAC-oncologia, módulo específico do SIA/SUS, de acesso irrestrito, disponível no sítio do DATASUS (www. datasus.gov.br), acessados em 11/12/2015 .

Foram selecionadas as autorizaçóes com código 1 , que são apresentadas na primeira competência. Portanto, foram excluídas as autorizaçóes com o mesmo número (código 2), válidas para a segunda e terceiras competências, evitando a redundância de informação em razão da reapresentação da APAC ao longo de sua validade. Não houve nenhuma ocorrência de APAC com código 3 (única).

Posteriormente, o banco foi restrito àqueles com diagnóstico de neoplasia maligna de mama (C50) e separados por Região do país, originando seis bancos (Brasil e Regióes), para o período de 2010 a 2014.

As seguintes variáveis foram consideradas: faixa etária em anos (menores de 40, 40 a 49, 50 a 69, 70, ou mais); estadiamento: (0, I, II, III, IV); Região do país em 
que o estabelecimento de saúde está situado; código de autorização da APAC; CNS: variável codificada, utilizada para individualizar os casos.

Em cada banco regional, com códigos de APAC iniciais, foram separadas as APAC com CNS inéditos (CNS que registraram apenas uma autorização para tratamento de quimioterapia) das APAC com CNS repetidos (CNS que registraram mais de uma autorizaçáo para tratamento de quimioterapia).

No grupo de APAC com CNS repetidos, foram criados dois subgrupos: a) CNS cujo estadiamento se manteve o mesmo em todas as APAC registradas; e b) CNS cujo estadiamento náo se manteve o mesmo entre as APAC registradas.

Verificaram-se a relação entre estadiamento e faixa etária e a frequência de alteração no estadiamento no grupo de APAC inicial com CNS repetido.

Para a limpeza do banco de dados e análise, foram utilizados o programa R versão 3.1.17 e o Excel 2010.

Como o estudo utilizou apenas dados secundários de acesso irrestrito, isenta-se da necessidade de submissão ao Conselho de Ética conforme parágrafo único da Resolução do Conselho Nacional de Saúde no 510/2016 .

\section{RESULTADOS}

No período entre 2010 a 2014, no Brasil, foram informadas no SIA/SUS 2.211.456 APAC iniciais para o tratamento de quimioterapia do câncer de mama no país, referentes a 280.379 registros de CNS, a maioria da Região Sudeste. Observou-se que 30.463 (1,4\%) autorizaçóes para quimioterapia foram para CNS que só apareceram uma vez (inéditos) e, consequentemente, só tinham uma APAC. As demais autorizações (2.180.993) foram para CNS, que apresentaram duas ou mais APAC nesse período (repetidos); neste caso, resultando uma razão de 8,7 APAC de quimioterapia para cada CNS. A Região Norte apresentou a menor razão APAC/CNS $(7,6)$ e a Sul, a maior $(9,1)$ (Tabela 1$)$.
Das 2.211.456 APAC, o estadiamento II foi o mais frequente em todas as Regióes, seguido do III, exceto na Região Sul, em que o estádio I foi o segundo mais frequente. Observa-se também que, embora a Região Sul tenha apresentado a maior proporção em estádio I (27,3\%), apresentou também a maior proporção no estádio IV (11,5\%). As Regiôes Norte e Nordeste tiveram os menores percentuais de tumores nos estádios 0 e I e os maiores percentuais de tumores nos estádios II e III. Essas duas Regiôes foram as que apresentaram a maior proporção de estádio avançado (III e IV), 42,7\% e 41,9\%, respectivamente. $\mathrm{O}$ estádio 0 variou de $0,2 \%$ na Regiáo Norte a 3,9\% na Região Centro-Oeste (Tabela 2).

$\mathrm{Na}$ análise entre o estadiamento e faixa etária, o estadiamento II foi o mais frequente em todas as faixas etárias, exceto entre as mulheres mais jovens, com menos de 40 anos, em que prevaleceu o estadiamento III. O estádio I aumentou com a idade, enquanto o estádio III diminuiu. O estádio IV apresentou maiores proporçôes em mulheres com menos de 40 anos e com 70 ou mais. No Brasil, o estadiamento avançado (III e IV) foi de 51,6\% na faixa etária menor de 40 anos e foi reduzindo com o aumento da idade. Nas Regiōes Norte e Centro-Oeste, observou-se que os estadiamentos mais recorrentes na faixa etária de 40 a 49 anos foram II e III. Destaca-se que a Região Sul, comparada às demais Regiōes, apresentou uma maior concentraçáo do estadiamento I na faixa etária de 50 a 69 anos (Tabela 2).

Entre os CNS que se repetiram $(n=249.619)$ e que necessitaram de mais de uma APAC para tratamento quimioterápico do câncer de mama, 18,1\% tiveram registros diferentes de estadiamento ao longo do período de estudo, tendo as Regióes Sul $(22,7)$ e Centro-Oeste $(18,9 \%)$ a maior proporção de CNS com estadiamentos diferentes registrados nas APAC. As Regiôes Norte e Sul registraram, respectivamente, a maior $(91,1 \%)$ e menor $(77,3 \%)$ proporção de CNS que náo tiveram o estadiamento alterado ao longo do período (Tabela 3).

Tabela 1. Distribuição de Autorização de Procedimentos de Alta Complexidade (APAC) inicial para quimioterapia de câncer de mama e de Cartão Nacional de Saúde (CNS), Brasil e Regiões, 2010-2014

\begin{tabular}{|c|c|c|c|c|c|c|c|c|c|c|c|c|}
\hline & \multicolumn{2}{|c|}{ Norte } & \multicolumn{2}{|c|}{ Nordeste } & \multicolumn{2}{|c|}{ Sudeste } & \multicolumn{2}{|c|}{ Sul } & \multicolumn{2}{|c|}{ Centro-Oeste } & \multicolumn{2}{|c|}{ Brasil } \\
\hline & N & $\%$ & N & $\%$ & N & $\%$ & N & $\%$ & N & $\%$ & N & $\%$ \\
\hline Total de APAC (a) & 50.915 & 2,3 & 450.611 & 20,4 & 1.088.322 & 49,2 & 509.383 & 23,0 & 112.225 & 5,1 & 2.211 .456 & 100,0 \\
\hline Total de APAC com CNS inéditos (b) & 1.175 & 3,9 & 6.342 & 20,8 & 14.737 & 48,4 & 6.068 & 19,9 & 2.141 & 7,0 & 30.463 & 100,0 \\
\hline Total de APAC com CNS repetidos (c) & 49.740 & 2,3 & 444.269 & 20,4 & 1.073 .585 & 49,2 & 503.315 & 23,1 & 110.084 & 5,0 & 2.180 .993 & 100,0 \\
\hline Total de CNS (d) & 7.726 & 2,8 & 58.864 & 21,0 & 136.211 & 48,6 & 61.389 & 21,9 & 16.189 & 5,8 & 280.379 & 100,0 \\
\hline Total de CNS com mais de I APAC (e) & 6.551 & 2,6 & 52.522 & 21,0 & 121.474 & 48,6 & 55.321 & 22,1 & 14.048 & 5,6 & 249.916 & 100,0 \\
\hline Razão entre (c) e (e) & \multicolumn{2}{|c|}{7,6} & \multicolumn{2}{|c|}{8,5} & \multicolumn{2}{|c|}{8,8} & \multicolumn{2}{|c|}{9,1} & \multicolumn{2}{|c|}{7,8} & \multicolumn{2}{|c|}{8,7} \\
\hline
\end{tabular}

Fonte. Sistema de Informação Ambulatorial (SIA/SUS) 6 . 
Tabela 2. Distribuição de Autorização de Procedimentos de Alta Complexidade (APAC) inicial em quimioterapia de câncer de mama (C50), por estadiamento e faixa etária, Brasil e Regiões, 2010-2014

\begin{tabular}{|c|c|c|c|c|c|c|c|}
\hline \multirow{2}{*}{ Regiões } & \multirow{2}{*}{ Estadiamento } & \multicolumn{4}{|c|}{ Faixa Etária (anos) } & \multicolumn{2}{|c|}{ Total de APAC } \\
\hline & & $\leq \mathbf{4 0} \%$ & 40 a $49 \%$ & 50 a $69 \%$ & $>70 \%$ & $\mathbf{n}$ & $\%$ \\
\hline \multirow{6}{*}{ Norte } & 0 & 0,1 & 0,2 & 0,2 & 0,1 & 80 & 0,2 \\
\hline & I & 9,5 & 11,7 & 17,0 & 21,0 & 7.946 & 15,6 \\
\hline & II & 32,9 & 40,2 & 43,2 & 43,3 & 21.156 & 41,6 \\
\hline & III & 47,5 & 40,1 & 31,5 & 24,5 & 17.305 & 34,0 \\
\hline & IV & 10,1 & 7,9 & 8,2 & 11,1 & 4.428 & 8,7 \\
\hline & Total & 100,0 & 100,0 & 100,0 & 100,0 & 50.915 & 100,0 \\
\hline \multirow{6}{*}{ Nordeste } & 0 & 0,9 & 1,1 & 1,3 & 1,5 & 5.750 & 1,3 \\
\hline & I & 9,8 & 14,1 & 18,3 & 20,4 & 76.851 & 17,0 \\
\hline & II & 34,4 & 39,4 & 39,8 & 41,0 & 178.132 & 39,3 \\
\hline & III & 42,8 & 35,8 & 30,6 & 25,4 & 142.821 & 31,5 \\
\hline & IV & 12,1 & 9,6 & 10,1 & 11,7 & 47.057 & 10,4 \\
\hline & Total & 100,0 & 100,0 & 100,0 & 100,0 & 452.687 & 100,0 \\
\hline \multirow{6}{*}{ Sul } & 0 & 2,2 & 2,1 & 2,2 & 2,3 & 11.286 & 2,2 \\
\hline & I & 18,1 & 25,1 & 29,1 & 27,1 & 139.018 & 27,3 \\
\hline & II & 33,3 & 35,6 & 34,8 & 35,4 & 178.317 & 35,0 \\
\hline & III & 33,5 & 27,5 & 22,6 & 21,3 & 122.132 & 24,0 \\
\hline & IV & 13,0 & 9,7 & 11,2 & 13,9 & 58.630 & 11,5 \\
\hline & Total & 100,0 & 100,0 & 100,0 & 100,0 & 509.383 & 100,0 \\
\hline \multirow{6}{*}{ Sudeste } & 0 & 3,0 & 2,7 & 3,0 & 3,4 & 33.042 & 3,0 \\
\hline & I & 13,1 & 20,1 & 24,9 & 25,4 & 254.731 & 23,4 \\
\hline & II & 32,0 & 36,6 & 36,5 & 36,4 & 394.764 & 36,3 \\
\hline & III & 39,2 & 30,9 & 25,6 & 21,9 & 289.270 & 26,6 \\
\hline & IV & 12,6 & 9,7 & 10,0 & 12,8 & 116.515 & 10,7 \\
\hline & Total & 100,0 & 100,0 & 100,0 & 100,0 & 1.088 .322 & 100,0 \\
\hline \multirow{6}{*}{ Centro-Oeste } & 0 & 4,7 & 4,0 & 3,9 & 3,6 & 4.407 & 3,9 \\
\hline & I & 13,8 & 17,2 & 21,7 & 23,0 & 22.656 & 20,2 \\
\hline & II & 30,8 & 36,8 & 39,3 & 41,2 & 42.996 & 38,3 \\
\hline & III & 42,0 & 36,2 & 28,6 & 24,8 & 34.614 & 30,8 \\
\hline & IV & 8,7 & 5,8 & 6,6 & 7,5 & 7.552 & 6,7 \\
\hline & Total & 100,0 & 100,0 & 100,0 & 100,0 & 112.225 & 100,0 \\
\hline \multirow{6}{*}{ Brasil } & 0 & 2,3 & 2,2 & 2,5 & 2,8 & 54.565 & 2,5 \\
\hline & I & 13,2 & 19,5 & 24,3 & 24,6 & 501.202 & 22,7 \\
\hline & II & 32,9 & 37,1 & 37,0 & 37,4 & 815.365 & 36,9 \\
\hline & III & 39,4 & 31,8 & 26,1 & 22,6 & 606.142 & 27,4 \\
\hline & IV & 12,2 & 9,4 & 10,1 & 12,6 & 234.182 & 10,6 \\
\hline & Total & 100,0 & 100,0 & 100,0 & 100,0 & 2.211 .456 & 100,0 \\
\hline
\end{tabular}

Fonte. Sistema de Informação Ambulatorial (SIA/SUS) ${ }^{6 .}$

Tabela 3. Distribuição de Cartão Nacional de Saúde (CNS) com mais de uma Autorização de Procedimentos de Alta Complexidade (APAC) inicial segundo status de mudança do estadiamento. Brasil e Regiões, 2010-2014

\begin{tabular}{|c|c|c|c|c|c|c|}
\hline \multirow[t]{2}{*}{ Brasil e Regiões } & \multicolumn{2}{|c|}{$\begin{array}{c}\text { CNS com mais de } \\
\text { uma APAC e que o } \\
\text { estadiamento não mudou }\end{array}$} & \multicolumn{2}{|c|}{$\begin{array}{l}\text { CNS com mais de } \\
\text { uma APAC e que o } \\
\text { estadiamento mudou }\end{array}$} & \multicolumn{2}{|c|}{$\begin{array}{c}\text { Total de CNS com mais de } \\
\text { Uma APAC }\end{array}$} \\
\hline & $\mathbf{N}$ & $\%$ & $\mathbf{N}$ & $\%$ & $\mathbf{N}$ & $\%$ \\
\hline Norte & 5.967 & 91,1 & 584 & 8,9 & 6.551 & 100,0 \\
\hline Nordeste & 44.178 & 84,1 & 8.344 & 15,9 & 52.522 & 100,0 \\
\hline Sudeste & 100.431 & 82,7 & 21.043 & 17,3 & 121.474 & 100,0 \\
\hline Sul & 42.790 & 77,3 & 12.531 & 22,7 & 55.321 & 100,0 \\
\hline Centro-Oeste & 11.387 & 81,1 & 2.661 & 18,9 & 14.048 & 100,0 \\
\hline Brasil & 204.753 & 81,9 & 45.163 & 18,1 & 249.916 & 100,0 \\
\hline
\end{tabular}

Fonte. Sistema de Informaçấo Ambulatorial (SIA/SUS) ${ }^{6}$. 
Entre os CNS que tiveram registros diferentes de estadiamento, a maioria variou entre dois registros de estadiamento e houve casos de variaçóes entre quatro e até cinco registros (Tabela 4).

$\mathrm{Na}$ distribuição do estadiamento em todas as Regiōes e no Brasil entre o grupo que reuniu todas as autorizaçóes de APAC (Fig.1a), observa-se que a Região Norte apresentou a menor porcentagens de APAC com estádio I e que o estádio 0 tem uma expressão maior nas Regiôes Sudeste e Centro-Oeste. Entre as APAC com CNS inédito (Fig.1b), as Regiōes Sudeste e Centro-Oeste apresentaram as maiores porcentagens de estádio 0 e a Regiáo Sul, a maior porcentagem de estádio I; contudo, em todas as Regióes, observou-se que a proporção de estádio IV foi em torno de $20 \%$. No grupo de CNS com mais de uma APAC em que o estadiamento não mudou (Fig.1c), destaca-se a Região Sul com a maior proporção de estádio I (30\%) e graficamente sem expressão do estádio 0. No grupo de CNS com mais de uma APAC em que o estadiamento mudou (Fig.1d), observou-se uma maior variação entre as Regióes quanto ao estadiamento informado, a Região Norte apresentou os menores registros de estadiamentos 0 e I e a maior proporção de estádio IV. Já a Região Sudeste apresentou a maior proporção de estádio 0 e a segunda maior proporção de estádio IV.

Tabela 4. Distribuição de Cartão Nacional de Saúde (CNS) com mais de uma Autorização de Procedimentos de Alta Complexidade (APAC) inicial segundo número de mudanças no estadiamento, Brasil e Regiões, 2010-2014

\begin{tabular}{|c|c|c|c|c|c|c|c|c|c|c|}
\hline \multirow{3}{*}{ Brasil e Regióes } & \multicolumn{8}{|c|}{ Número de mudanças no estadiamento } & \multirow{2}{*}{\multicolumn{2}{|c|}{$\begin{array}{l}\text { Total CNS com mais } \\
\text { de uma APAC, nos } \\
\text { quais houve mudançc } \\
\text { no estadiamento }\end{array}$}} \\
\hline & \multicolumn{2}{|c|}{2 vezes } & \multicolumn{2}{|c|}{3 vezes } & \multicolumn{2}{|c|}{4 vezes } & \multicolumn{2}{|c|}{5 vezes } & & \\
\hline & $\mathbf{N}$ & $\%$ & $\mathbf{N}$ & $\%$ & $\mathbf{N}$ & $\%$ & $\mathbf{N}$ & $\%$ & $\mathbf{N}$ & $\%$ \\
\hline Norte & 553 & 94,7 & 29 & 5,0 & 2 & 0,3 & 0 & 0,0 & 584 & 100,0 \\
\hline Nordeste & 7.047 & 84,5 & 1.084 & 13,0 & 193 & 2,3 & 20 & 0,2 & 8.344 & 100,0 \\
\hline Sudeste & 18.536 & 88,1 & 2.292 & 10,9 & 205 & 1,0 & 10 & 0,0 & 21.043 & 100,0 \\
\hline Sul & 9.644 & 77,0 & 2.264 & 18,1 & 546 & 4,4 & 77 & 0,6 & 12.531 & 100,0 \\
\hline Centro-Oeste & 2.204 & 82,8 & 405 & 15,2 & 47 & 1,8 & 5 & 0,2 & 2.661 & 100,0 \\
\hline Brasil & 37.984 & 84,1 & 6.074 & 13,4 & 993 & 2,2 & 112 & 0,2 & 45.163 & 100,0 \\
\hline
\end{tabular}

Fonte. Sistema de Informação Ambulatorial (SIA/SUS) ${ }^{6}$.
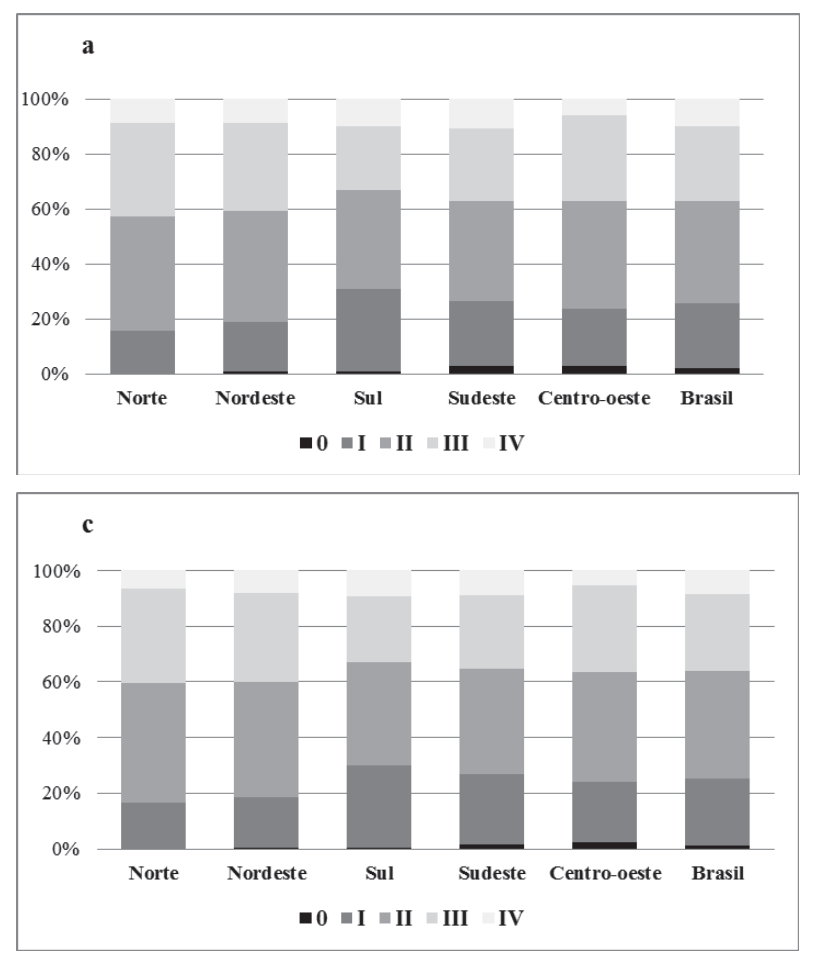
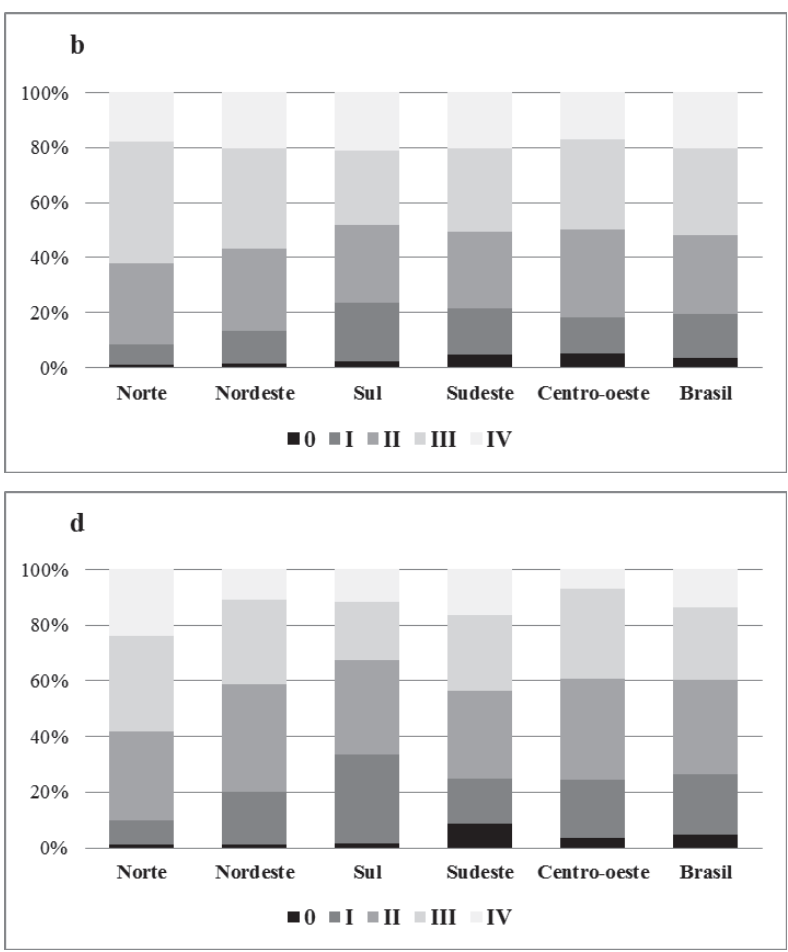

Figura 1. Distribuição percentual do estadiamento, Brasil e Regiões, 2010-2014, de acordo com: (a) total de Autorizações de Procedimento de Alta Complexidade (APAC) inicial; (b) total de APAC com Cartão Nacional de Saúde (CNS) inédito; (c) total de CNS com mais de uma APAC sem mudança de estádio; (d) total de CNS com mais de uma APAC com mudança de estádio Fonte: Sistema de Informaçáo Ambulatorial (SIA/SUS) ${ }^{6}$. 


\section{DISCUSSÃO}

Como foram consideradas no estudo apenas as APAC iniciais, não houve $\mathrm{APAC}$ com código de autorização igual assegurando que cada CNS estivesse associado às autorizaçôes distintas. Sendo assim, foi possível verificar o total de CNS com mais de uma APAC para o tratamento do mesmo tumor possibilitando estimar o tempo médio de tratamento. Dessa forma, tendo a APAC uma validade de três meses ${ }^{3}$, o tempo de tratamento de quimioterapia para câncer de mama no país seria em torno de 26 meses. A Região Norte apresentou o menor tempo (22 meses) e a Região Sul o maior tempo (27 meses). Uma hipótese que possa explicar o tempo médio de tratamento maior na Região Sul é o fato de esta apresentar a maior proporção de casos em estádio iniciais justificando a necessidade de um maior número de APAC; situação oposta à da Regiâo Norte.

A informação do tempo médio para tratamento de quimioterapia pode subsidiar a organizaçáo dos estabelecimentos habilitados em oncologia, bem como o monitoramento dos tratamentos fora domicílio.

A avaliação do estadiamento do câncer de mama, a partir dos dados do registro Hospitalar de Câncer (RHC), entre 2010-2011, mostrou que os estádios II e III foram os mais frequentes ${ }^{9}$, sendo consistente com achados do presente estudo, no qual os estadiamentos II e III foram prevalentes no país e em todas as Regiôes, com exceção da Região Sul. Vale destacar que o RHC reúne todos os casos atendidos nas unidades habilitadas independente da modalidade terapêutica e, uma vez que quimioterapia é o tratamento de escolha na maioria ${ }^{3,10}$ dos casos de câncer de mama, é admissível que os estádios observados sejam equivalentes entre esses sistemas de informação.

O estudo verificou que, apesar de a maior proporçáo de autorizaçóes iniciais na APAC serem estádio II, os estádios avançados (III e IV) são mais frequentes nas Regiōes Norte e Nordeste quando comparados ao valor médio observado no Brasil. Estudo realizado para o país, referente ao período de 1980 a 2006, mostrou reduçáo da mortalidade por câncer de mama em todas as capitais do país, exceto nas da Região Norte ${ }^{11}$. Ainda que tenham sido estudados períodos diferentes e que a mortalidade seja determinada por vários fatores, os achados do presente estudo demonstram que ainda se observa um quadro desfavorável à deteç̧ấo precoce do câncer de mama na Região Norte. Destaca-se ainda que a Regiáo Norte, com maior proporção de casos avançados, foi a com menor razão de APAC por CNS.

Mulheres mais jovens e mulheres mais idosas apresentaram maior proporção de estádios IV no Brasil e em todas as Regiôes. Esse achado precisa ser melhor investigado, mas uma possível explicação pode ser a natureza do câncer de mama nas mulheres mais joven $s^{12}$, que sendo mais agressivos evoluem rapidamente e não se beneficiam das estratégias de detecção precoce; enquanto, nas idosas, pode ser em razão da dificuldade de acesso aos serviços de saúde ou falhas no programa de detecção precoce do câncer de mama. Estudo realizado em serviço de referência da Saúde da Mulher em São Paulo, utilizando os seus dados do $\mathrm{RHC}^{13}$, destaca o desafio de conseguir acesso aos serviços de saúde ainda em fase inicial da doença, ainda que tenha encontrado em seus achados maior proporçấo de mulheres idosas em estádios II e III.

Muito embora o manual da APAC seja claro quanto à manutenção do estadiamento clínico ao longo do tratamento, o estudo revelou que em $18 \%$ dos casos o estadiamento foi modificado, corroborando os achados de Gadelha et al. ${ }^{14}$ para câncer de mama no Estado do Rio de Janeiro, que identificaram mudança no estadiamento da APAC ao longo do tempo para o período entre 1999 e 2003. Esses autores ressaltaram que mudanças no percurso da doença como: estabilidade, progressão ou ainda recidiva são previstas e o procedimento terapêutico e sua finalidade devem estar atrelados a esse percurso; mas, ainda assim, o estadiamento deve permanecer imutável ${ }^{14}$.

Entende-se que, para o monitoramento da evoluçáo do caso durante o tratamento, seria interessante dispor da informação do estadiamento em diferentes momentos. Contudo, isso náo está previsto no manual da APAC. Sendo assim, não se pode afirmar se a mudança no registro do estadiamento reflete um erro de informação ou a evolução da doença.

As informaçóes disponíveis na APAC-onco, quando de qualidade, têm o potencial de auxiliar no monitoramento dos casos de câncer no país, bem como dos estabelecimentos habilitados para o tratamento em oncologia. Por conseguinte, o estudo ressalta a importância de se primar pelo treinamento dos responsáveis pelo sistema de informação com vistas a reduzir incongruências.

Entre as limitaçôes identificadas no presente estudo, não foi possível avaliar a direçáo da mudança de estadiamento no grupo com mesmo CNS e com um ou mais estádios informados. Além disso, o estudo não analisou o tempo médio de tratamento por estadiamento clínico, o que poderia ter fornecido diferentes estimativas de tempo.

\section{CONCLUSÃO}

O estadiamento clínico é fundamental para definição terapêutica e para avaliar as açóes de diagnóstico precoce, sendo, portanto, necessário zelar pela qualidade dessa informação. A análise, a partir da APAC de quimioterapia de mama, demonstrou que há mudança no estádio, em parte 
possivelmente relacionada à evoluçáo da doença. Contudo, diante do que está previsto no manual da APAC, não é possível desconsiderar erro de preenchimento. Assim sendo, entende-se que uma avaliação do instrumento de registro da APAC possibilitaria o refinamento da informação.

Ainda que os sistemas de informação do Ministério da Saúde possuam uma finalidade administrativa de repasse financeiro, a APAC-oncologia tem dados extremamente úteis para o planejamento da rede de atenção à saúde no que tange ao tratamento oncológico. Estimular a sua utilização pelos gestores como ferramenta de planejamento deve ser um norte para os vários atores envolvidos na discussão sobre a melhoria da atençấo oncológica no Brasil.

\section{CONTRIBUIÇÕES}

Adriana Tavares de Moraes Atty contribuiu na concepção e delineamento do estudo, extração, análise e interpretação dos dados, redação e revisão crítica relevante do conteúdo intelectual do manuscrito. Jeane Glaucia Tomazelli colaborou na análise e interpretação dos dados; redação e revisão crítica relevante do conteúdo intelectual do manuscrito. Maria Beatriz Kneipp Dias colaborou na análise e interpretação dos dados e na revisão crítica relevante do conteúdo intelectual do manuscrito. Todos os autores aprovaram a versão final do manuscrito e declaram ser responsáveis por todos os aspectos do trabalho, garantindo sua precisão e integridade.

\section{DECLARAÇÃO DE CONFLITO DE INTERESSES}

As autoras declaram potencial conflito de interesses pelos vínculos empregatícios com o INCA.

\section{REFERÊNCIAS}

1. American Cancer Society. Global Cancer Facts \& Figures. 3th. ed. [Internet]. Atlanta; 2015. [acesso em 2018 jan 9]. Disponível em: https://www.cancer.org/content/ $\mathrm{dam} /$ cancer-org/research/cancer-facts-and-statistics/ global-cancer-facts-and-figures/global-cancer-facts-andfigures-3rd-edition.pdf

2. Ministério da Saúde (BR). Portaria GM/MS no 874, de 16 de maio de 2013. Institui a Política Nacional para a Prevençáo e Controle do Câncer na Rede de Atençáo à Saúde das Pessoas com Doenças Crônicas no âmbito do
Sistema Único de Saúde (SUS). Brasília (DF): Diário Oficial da União. Seção I (2013. maio 29); 2013.

3. Ministério da Saúde (BR). Manual de bases técnicas da oncologia: Sistema de informaçôes ambulatoriais (SIA/ SUS). 20ª . ed. Brasília, DF: Ministério da Saúde; 2015.

4. Instituto Nacional de Câncer José Alencar Gomes da Silva. TNM: classificaçấo de tumores malignos. $7^{\mathrm{a}} \mathrm{ed}$. Rio de Janeiro: INCA; 2012.

5. Ministério da Saúde (BR). Sistemas de Informação da Atenção à Saúde. Brasília, DF: Ministério da Saúde; 2015.

6. Ministério da Saúde (BR). Sistemas de Informaçôes Ambulatoriais do SUS. [Internet]. Rio de Janeiro: Ministério da Saúde; 2015. [acesso em 2015 dez 11]. Disponível em: http://sia.datasus.gov.br/principal/index.php

7. R Core Team. R: a language and environment for statistical computing [Internet]. Vienna: R Foundation for Statistical Computing; 2013. [acesso em 2018 jan 9]. Disponível em: http://www.R-project.org/

8. Ministério da Saúde (BR). Resolução do CNS no 510, de 7 de abril de 2016. Brasília (DF): Diário Oficial da União. Seção I, fls. 44-6 (2016 maio 24); 2016. Disponível em: http://conselho.saude.gov.br/resolucoes/2016/reso510.pdf

9. Instituto Nacional de Câncer José de Alencar Gomes da Silva. Informativo Detecção Precoce [Internet].2015;6(3). [acesso em 2018 jan 9]. Disponível em: http://wwwl.inca. gov.br/inca/Arquivos/informativo_numero3_2015.pdf

10. Ministério da Saúde (BR). Portaria SAS no 1008, de 30 de setembro de 2015. Aprova as Diretrizes Diagnósticas e Terapêuticas do Carcinoma de Mama. Brasília (DF): Diário Oficial da Uniáo. (2015 set 30); 2015.

11. Silva GA, Gamarra CJ, Girianelli VR, Valente JG. Tendência da mortalidade por câncer nas capitais e interior do Brasil entre 1980 e 2006. Rev Saude Publica. 2011;45(6):1009-18.

12. Clagnan WS, Andrade JM, Carrara HHA, Tiezze DG, Reis FJC, Marana HCR, Abrão RA. Idade como fator independente de prognóstico no câncer de mama. Rev Bras Ginecol Obstet. 2008;30(2):67-74.

13. Souza CB, Fustinoni SM, Amorim MHC, Zandonade E, Matos JC, Schirmer J. Estudo do tempo entre o diagnóstico e o início do tratamento do câncer de mama em idosas de um hospital de referência em São Paulo, Brasil. Ciênc. saúde coletiva. 2015;20(12):3805-3816.

14. Gadelha MIP, Costa MR, Almeida RT. Estadiamento de tumores malignos: análise e sugestôes a partir de dados da APAC. Rev Bras Cancerol. 2005;51(3):193-9. 


\begin{abstract}
Introduction: Radiotherapy and chemotherapy are therapeutic modalities for the treatment of cancer. In Brazilian public health system, they require the High Complexity Ambulatory Procedure Authorization (APAC), which has important information about patients and health services. This study aims to do an exploratory analysis of breast cancer stage information. Method: It's an exploratory and descriptive study on staging of breast cancer in Brazil and regions, reported in the APAC of chemotherapy, from january 2010 to december 2014. The variables that were considered are: age group, staging, authorization code of APAC, national health card (CNS) and region of the country where the health service is located. Data were obtained through DATASUS's homepage. Results: There were 2,211,456 first APAC referring to 280,379 CNS. APAC with repeated CNS were 98,6\% and the ratio between APAC/CNS was 8,7. Of these, $18.1 \%$ had staging changes. Conclusion: The clinical staging established at the beginning of treatment should stay unchanged throughout patient's life, according to the recommended norm of APAC. However, it would be of great value to monitor the cases if it were possible to take notes about the progression of the disease and consequently the staging. It was not possible to state whether the observed changes in staging represented disease evolution or information error. Since APAC is a useful source of information for the planning and monitoring of health services, it is important to prioritize the quality of the information without denying the need to improve the system.
\end{abstract}

Key words: Neoplasm Staging; Unified Health System; Health Information Systems.

\title{
Resumen
}

Introducción: La radioterapia y la quimioterapia, modalidades terapéuticas para el tratamiento del cáncer en el sistema público de salud, exigen liberación de la Autorización de Procedimiento Ambulatorial de Alta Complejidad (APAC), la cual reúne informaciones importantes sobre los pacientes y sobre los establecimientos de salud. El objetivo de este estudio fue realizar un análisis exploratorio de las informaciones sobre estadio de los casos de cáncer de mama. Método: el estudio exploratorio, descriptivo, sobre la estadificación de cáncer de mama, en Brasil y regiones, informados en las APAC de quimioterapia, en el período de enero de 2010 a diciembre de 2014. Fueron consideradas las variables: grupo de edad, estadificación, código de autorización de la APAC, tarjeta nacional de salud y región del país del establecimiento de salud. Los datos fueron obtenidos a través de DATASUS. Resultados: Hubo 2.211 .456 primer APAC referente a 280.379 CNS. APAC con repetición del SNC fue del 98,6\% y la relación APAC/SNC fue de 8,7. De éstos, 18,1\% tenían cambios de estadificación. Conclusión: La estadificación clínica establecida al inicio del tratamiento debería permanecer inalterada a lo largo de la vida del paciente, según la norma que orienta el llenado de la APAC. Sin embargo, sería de gran valía al monitoreo de los casos si fuera posible registrar el avance de la enfermedad y, consecuentemente de la estadificación. No fue posible afirmar si los cambios observados en la estadificación representaban evolución de la enfermedad o error de información. Siendo APAC una fuente de información útil a la planificación y al monitoreo de los servicios de salud vale primar por la calidad de la información sin negar la necesidad de perfeccionamiento del sistema.

Palabras clave: Estadificación de Neoplasias; Sistema Único de Salud; Sistemas de Información en Salud. 